Research paper

\title{
Canine neutrophils activate effector mechanisms in response to Leishmania infantum
}

\author{
Maria Pereira $^{\mathrm{a}, \mathrm{b}}$, Ana Valério-Bolas ${ }^{\mathrm{a}, 1}$, David Santos-Mateus ${ }^{\mathrm{a}, 1}$, Graça Alexandre-Pires ${ }^{\mathrm{c}}$, \\ Marcos Santos $^{\mathrm{c}}$, Armanda Rodrigues ${ }^{\mathrm{c}}$, Hugo Rocha ${ }^{\mathrm{d}}$, Ana Santos ${ }^{\mathrm{d}}$, Catarina Martins ${ }^{\mathrm{e}}$, Ana Tomas ${ }^{\mathrm{f}}$, \\ Filipe Passero $^{g}$, Isabel Pereira da Fonseca ${ }^{c}$, Gabriela Santos-Gomes ${ }^{\mathrm{a} \text {, }}$ \\ a Global Health and Tropical Medicine, GHMT, Instituto de Higiene e Medicina Tropical, IHMT, Universidade Nova de Lisboa, UNL, Rua da Junqueira 100, 1349-008 \\ Lisbon, Portugal \\ b Polytechnic Institute of Portalegre, Higher Agrarian School of Elvas, Edifício do Trem Auto, Avenida 14 de Janeiro s/n Apartado 254, 7350-903 Elvas, Portugal \\ ${ }^{\mathrm{c}}$ Interdisciplinary Animal Health Research Centre, Faculty of Veterinary Medicine, University of Lisbon, Av. Universidade Técnica, 1300-477 Lisbon, Portugal \\ d Division of Veterinary Medicine, National Guard, Largo do Carmo 1200-92 Lisbon, Portugal \\ e Chronic Diseases Research Center, Immunology, NOVA Medical School, New University of Lisbon, Rua Câmara Pestana n 6, 6-A Edifício CEDOC II, 1150-082 Lisbon, \\ Portugal \\ ${ }^{\mathrm{f}}$ I3S, Instituto de Investigação e Inovação em Saúde. IBMC, Instituto de Biologia Molecular e Celular and ICBAS, Instituto de Ciências Biomédicas Abel Salazar, University \\ do Porto, Rua Alfredo Allen, 208, 4200-135 Porto, Portugal \\ ${ }^{g}$ São Paulo State University (Unesp), Institute of Biosciences, São Vicente, Praça Infante Dom Henrique, S/N, 11330-900 São Vicente, SP, Brazil
}

\section{A R T I C L E I N F O}

\section{Keywords:}

Canine leishmaniosis

Neutrophils

Superoxide

Neutrophil elastase

Neutrophil extracellular traps

Electron microscopy

\begin{abstract}
A B S T R A C T
Canine leishmaniosis caused by $L$. infantum is a severe zoonotic disease. Although macrophages are the definitive host cells, neutrophils are the first cells to encounter the parasite soon after its inoculation in the dermis by the phlebotomine vector. To study the interaction of dog neutrophils and L. infantum promastigotes, blood neutrophils were isolated from healthy donors and the infection was established in vitro. In the majority of the dogs, L. infantum was efficiently phagocytized by neutrophils, and oxidative (superoxide production) and non-oxidative (neutrophil elastase exocytosis) intracellular effector mechanisms were activated, but the release of neutrophil extracellular traps was minimized. Furthermore, promastigotes and culture supernatants induced neutrophil migration, but the prior contact with Leishmania inhibits chemotaxis, which might contribute to neutrophil retention at the inoculation site. Neutrophil-parasite interaction resulted in a decrease in parasite viability, although some intracellular promastigotes survive and maintain their proliferative capacity. These findings indicate that dog neutrophils are competent effector cells able to control the initial $L$. infantum infection. However, some parasites evade intracellular effector mechanisms and can be transferred to the definitive host cell, the macrophage, contributing to the development of canine leishmaniosis.
\end{abstract}

\section{Introduction}

Adaptive immune response is crucial for the resolution of Leishmania infection. However, innate immune mechanisms have been gained importance, in particular the activity of neutrophils (or polymorphonuclear cells, PMN) as effector and immunomodulatory cells (Faria et al., 2012; Carlsen et al., 2015; Hurrell et al., 2016). PMN rapidly reach Leishmania injection site (Pompeu et al., 1991; Beil et al., 1992; Santos-Gomes et al., 2000; Matte and Olivier, 2002; Thalhofer et al., 2011; Ribeiro-Gomes and Sacks, 2012), recruited by parasite-, host- and sand fly-derived chemotactic factors (Hurrell et al., 2016), and seems to be the first cells to internalize the parasite (Ribeiro-Gomes et al., 2012; Hurrell et al., 2015).

Despite the high microbicidal potential of PMN, represented by the presence of granules full of enzymes and antimicrobial molecules and by the ability to produce reactive oxygen species (ROS), some internalized promastigotes survive (van Zandbergen et al., 2004). Indeed, promastigote uptake via lysosome-independent pathway leads to the formation of tight phagosome and promotes parasite survival, while internalization through lysosome-dependent pathway generates large phagosome that kills the parasite (Gueirard et al., 2008). Furthermore, some Leishmania species seem to be able to modulate PMN apoptosis,

\footnotetext{
* Corresponding author.

E-mail address: santosgomes@ihmt.unl.pt (G. Santos-Gomes).

${ }^{1}$ These authors contributed equally to this paper.
} 
prolonging cell life span or accelerating cell death, which could promote parasite transference to macrophages that reaches inoculation site later on (Aga et al., 2002; Gueirard et al., 2008; Carlsen et al., 2013; Falcão et al., 2015).

Neutrophil release Neutrophil Extracellular Traps (NET) in response to $L$. amazonensis, $L$. donovani, $L$. infantum, $L$. major, $L$. donovani and $L$. mexicana (es-Costa et al., 2009, 2014; es-Costa et al., 2009, 2014; Gabriel et al., 2010; Hurrell et al., 2015). However, some parasite species are protected from NET-mediated killing by lipophosphoglycan (LPG) (Gabriel et al., 2010), enzyme 3'-nucleotidase/nuclease (Guimarães-Costa et al., 2014) or by other factors not yet identified (Hurrell et al., 2015). Beyond microbicide and limiting spread effects, NET may also contribute to modulate the host immune system towards a pro-inflammatory response (Luo et al., 2014).

Thus, PMN seem to have a protective or a permissive impact at the early phase of Leishmania infection, depending on parasite species and host genetic background (Hurrell et al., 2016). Since innate immune mechanisms developed by the dog in the context of a L. infantum infection are largely unknown, this study aims to characterize the effector functions developed by peripheral blood PMN when in contact with $L$. infantum virulent promastigotes, investigating its contribution to the control of infection or, by the contrary, to disease progression.

\section{Material and methods}

\subsection{Experimental design}

To analyze neutrophil- $L$. infantum interactions during an early infection, blood PMN were isolated from 10 healthy dogs and cell purity and viability were determined. Then cells were in vitro exposed to virulent promastigotes and PMN effector functions were evaluated. The effect of parasite in PMN migration was assessed by a chemotaxis assay and the activation of intracellular (oxidative burst) and extracellular (granule exocytosis and NET emission) microbicide mechanisms were evaluated. Superoxide production was estimated by a colorimetric assay, exocytosis of neutrophil elastase (NE) was determined through a colorimetric enzyme/substrate reaction, NET structures were observed by electron microscopy and the exclusion of DNA and histone (NET constituents) from PMN nucleus were followed by fluorescent microscopy. The physical contact of PMN with promastigotes was characterized by flow cytometry, optical and electron microscopy and, the parasite fate tracked. Furthermore, the parasite effect on PMN natural viability was also evaluated by flow cytometry, investigating possible parasite modulation of PMN life span.

\subsection{Selection of healthy dogs}

A group of apparently healthy dogs belonging to Grupo de Intervenção Cinotécnico da Guarda Nacional Republicana and to Faculdade de Medicina Veterinária, Universidade de Lisboa (FMV/ ULisboa) was selected. Dogs were subjected to a complete physical examination and blood collection for a complete blood count, biochemical profile, ionogram and proteinogram to confirm their health status and the absence of vector borne diseases. Detection of antiLeishmania antibodies by indirect immunofluorescence assay (Kit Leishmania Spot IF', BioMerieux, France) with a cut off of 1:80, and blood and lymph node evaluation by q-PCR (Helhazar et al., 2013) was used to exclude $L$. infantum infection. The absence of Dirofilaria immitis microfilariae was confirmed by Knott test. Other hemoparasites, such as Babesia spp., Ehrlichia spp., Anaplasma spp. and Ricketsia spp. were ruled out by microscopic observation of blood smears and q-PCR. In addition, the presence of Mycoplasma spp. was evaluated by microscopic observation of blood smear. Ten clinically and analytically healthy dogs were chosen. Sample size was calculated on the basis of decided sample size, taking into account statistical significance and the minimum use of animals (Charan and Biswas, 2013). These dogs aged between 2 and 4 years $(2.5 \pm 0.9)$, weigh between 24 and $40 \mathrm{~kg}$ (32.3 \pm 5.2) and included pure breeds (1 Rafeiro do Alentejo Portuguese breed, 3 German Shepherd, and 1 Belgian Shepherd) and mixed breeds (5) of which four were females and six were males (Supplementary data 1 ).

Before inclusion in this study, dog's tutors were informed about the objective and the nature of this work and official consents were obtained. The study is in conformity with the institutional guidelines and the EU requirements and was approved by the Ethics and Welfare Committee of FMV/ULisboa.

\subsection{Parasites}

L. infantum zymodeme MON-1 (MHOM/PT/89/IMT151) virulent promastigotes collected from the stationary phase of growth of subcultures with less than five passages (Santos-Gomes and Abranches, 1996) and parasites expressing green fluorescent protein (GFP) (Marques et al., 2015) were used for PMN infection. Parasite concentration was assessed in a Neubauer-counting chamber (Marienfeld, Germany).

\subsection{PMN isolation and purification}

Each time, $20 \mathrm{~mL}$ of blood were collected into tubes containing citrate phosphate dextrose adenine (CPDA)-1 solution as anticoagulant (Kawasumi, Germany). PMN isolation was achieved using a Histopaque double-density gradient, according to the technique described by Strasser et al. (1998) and contaminant red blood cells were eliminated by osmotic lysis. Cells were resuspended in Hanks' Balanced Salt Solution (HBSS) (Sigma-Aldrich, USA) and its viability and concentration assessed by trypan blue exclusion in a Neubauer-counting chamber. Cell purity was determined by flow cytometry analysis and microscopic observation (Supplementary data 2).

\subsection{In vitro infection}

Dog PMN (5 $\times 10^{5}$ cells/well) were seeded in 96-well plates (Nunc, Denmark) with $L$. infantum or $L$. infantum-GFP promastigotes at a ratio parasite-PMN of $5: 1$ in $300 \mu \mathrm{L}$ of HBSS supplemented with $5 \%$ heatinactivated fetal bovine sera (FBS) (v/v). Plates were incubated at $37{ }^{\circ} \mathrm{C}$ in a humidified atmosphere containing $5 \%$ of $\mathrm{CO}_{2}$ for $1.5 \mathrm{~h}$ and $3 \mathrm{~h}$. In parallel, PMN cultures were also established to be used as negative controls. Infected and uninfected cultures were used in the following experiments.

\subsection{Interaction between $L$. infantum promastigotes and PMN}

The interaction between L. infantum-GFP parasites and PMN was evaluated by flow cytometry. After $1.5 \mathrm{~h}, 3 \mathrm{~h}$ and $4.5 \mathrm{~h}$ of incubation, resting-PMN (control) and PMN exposed to $L$. infantum-GFP were acquired by flow cytometry. $L$. infantum-GFP parasites were also acquired. The frequency of PMN-parasite association was assessed on one parameter histogram, plotting as FL1-H/GFP vs the number of events. Additionally, the contact between parasites and PMN was observed under an optical microscope (OM).

\subsection{Chemotaxis assay}

The migratory ability of resting-PMN and of PMN previously exposed to $L$. infantum was evaluated in a 96-well modified Boyden chamber (Neuroprobe Inc., USA) containing a polycarbonate $3 \mu \mathrm{m}$ pore membrane. Wells of the lower compartment were filled with $29 \mu \mathrm{L}$ of medium containing $5 \times 10^{5} \mathrm{~L}$. infantum promastigotes, $29 \mu \mathrm{L}$ of Leishmania chemotactic factor (LCF) (van Zandbergen et al., 2002) (positive control) or HBSS (negative control). In the upper compartment of the chamber, $25 \mu \mathrm{L}$ of resting-PMN and PMN-L. infantum 
cultures incubated for $1.5 \mathrm{~h}$ were added. The chamber was incubated for $60 \mathrm{~min}$ at $37^{\circ} \mathrm{C}$ in a humidified atmosphere containing $5 \% \mathrm{CO}_{2}$. PMN migration from the upper to the lower compartment was assessed in a Neubauer-counting chamber under OM.

\subsection{Superoxide production}

$\mathrm{O}_{2}{ }^{-}$production was measured using a colorimetric nitroblue tetrazolium (NBT) assay (Marques et al., 2015). Absorbance was measured at $570 \mathrm{~nm}$ using a microplate reader (Anthos 2010, Austria). RestingPMN were used as negative control and, phorbol myristate acetate (PMA, $0.2 \mu \mathrm{g} . \mathrm{mL}^{-1}$ ) (VWR, International) stimulated PMN and PMAstimulated PMN exposed to parasites were considered positive controls. Cell free-wells were used as a blank.

\subsection{Neutrophilic elastase (NE) exocytosis}

NE exocytosis was measured in culture supernatants of resting-PMN (negative control), PMN stimulated with $1 \mu \mathrm{g} \cdot \mathrm{mL}^{-1}$ of Escherichia coli lipopolysaccharide (LPS) (Sigma-Aldrich) (positive control) and of PMN exposed to $L$. infantum promastigotes, using a colorimetric reaction (Marques et al., 2015) that was quantified at $405 \mathrm{~nm}$ in a plate reader (TRIADTM 1065, DYNEX Technologies, EUA) at $\approx 0$ min (right away following the addition of substrate to the supernatants), $15 \mathrm{~min}$ and $30 \mathrm{~min}$ of incubation at $37^{\circ} \mathrm{C}$ in a humidified atmosphere containing $5 \%$ of $\mathrm{CO}_{2}$. It was assumed that enzyme activity is directly proportional to color intensity.

\subsection{Neutrophil extracellular traps (NET) release}

Resting-PMN (negative control), PMN incubated with $L$. infantum promastigotes, PMN stimulated with $1 \mu \mathrm{g} \cdot \mathrm{mL}^{-1}$ of PMA (positive control) and PMA-stimulated PMN exposed to L. infantum promastigotes (positive control) adhered to coverslips were incubated in HBSS $2 \%$ FBS and prepared for scanning electron microscopy (SEM) and immunofluorescence, according to Brinkmann et al. (2010). SEM samples were dried using the critical point drying method, coated with gold palladium and mounted on stubs. Cells were then observed under a scanning electronic microscope (JEOL 5200-LV) and images were acquired. The number of NET-generating cells was counted in 50 PMN of each condition. Furthermore, the orientation of promastigotes bounded to PMN (flagellum, aflagellar pole, or by any other place) was evaluated in 50 interactions. Immunofluorescence samples were stained with histone H1 (AE-4) FITC antibody (Santa Cruz Biotechnology, Germany) and DAPI, and observed under a fluorescence microscope (Nikon Eclipse 90i).

\subsection{PMN cell death}

Cell viability, apoptosis and necrosis were assessed by flow cytometry analysis. Resting-PMN (negative control), PMN exposed to $L$. infantum promastigotes and PMN stimulated with $100 \mu \mathrm{g} \cdot \mathrm{mL}^{-1}$ of (S)(+)-camptothecin (Sigma-Aldrich) (Campto, apoptotic positive control) were incubated in HBSS $10 \%$ FBS. Then, cultures were incubated with the commercial kit TACSTM Annexin V-FITC (R \& D Systems, USA), according with the manufacturer's instructions. Prior to the flow cytometry acquisition, cells were treated with $10 \mu \mathrm{L}$ of propidium iodine (PI, R \& D Systems). Untreated and annexin V-FITC or PI treated cells were used to compensate for PMN autofluorescence and for the fluorochrome fluorescence overlapping emission spectra. Resting untreated-PMN were used to delimit the quadrants for the evaluation of annexin V-FITC ${ }^{-} / \mathrm{PI}^{-}$(viable cells), annexin $\mathrm{V}^{-\mathrm{FITC}^{+}} / \mathrm{PI}^{-}$(apoptotic cells) and annexin V-FITC ${ }^{+/-} / \mathrm{PI}^{+}$cells (total necrotic cells) populations in a FL1-H (Annexin V-FITC) vs FL2-H (PI) dot-plot. Necrotic cells were also subdivided into annexin $\mathrm{V}-\mathrm{FITC}^{-} / \mathrm{PI}^{+}$(primary necrotic cells) and annexin V-FITC ${ }^{+} / \mathrm{PI}^{+}$(secondary necrotic cells).

\subsection{Viability of $L$. infantum promastigotes after PMN contact}

To study the impact of $L$. infantum exposition to dog PMN, parasite viability was assessed. L. infantum-PMN cultures were incubated for $2 \mathrm{~h}$. Then, $300 \mu \mathrm{L}$ of culture were seeded in a 24-wells plate containing $300 \mu \mathrm{L}$ per well of Schneider medium (Sigma-Aldrich) supplemented with 10\% FBS (complete SCHN) and two successive dilutions (1:4) were made. Cultures were incubated at $24^{\circ} \mathrm{C}$ for $24 \mathrm{~h}$. In parallel, cultures of L. infantum promastigotes were used as positive control. Viable promastigotes (moving parasites) were counted in a Neubauer-counting chamber.

\subsection{Ultrastructural PMN-parasite interrelation}

PMN-parasite ultrastructural interrelation was investigated by transmission electron microscopy (TEM). Cells exposed to promastigotes were centrifuged $(300 \times g$ for $10 \mathrm{~min}$ ), fixed in PBS (SigmaAldrich) $2 \%$ glutaraldehyde (Sigma-Aldrich) and $0.1 \%$ tannic acid (Sigma-Aldrich) for $1 \mathrm{~h}$ at $4{ }^{\circ} \mathrm{C}$ and then, pellets were washed two times in PBS. Samples were then prepared according to Yamamoto et al. (2015) and images were acquired under a transmission electron microscope (Jeol 1010).

\subsection{Viability of PMN-phagocytized parasites}

After $3 \mathrm{~h}$ of incubation, extracellular promastigotes were removed from parasite-exposed PMN by positive selection, using MicroBeads conjugated with monoclonal rat anti-mouse/human CD11b (Mac-1 $\alpha$ ) antibodies (Miltenyl Biotec, Germany) according to manufacturer's instructions. A previous experiment showed that this antibody also binds dogs PMN, allowing the separation between PMN and extracellular parasites. Viability and concentration of PMN free of extracellular parasites were assessed by trypan blue staining using a Neubauercounting chamber. Then, $5 \times 10^{5} \mathrm{PMN}$ were resuspended in $300 \mu \mathrm{L}$ of complete SCHN medium and incubated at $24^{\circ} \mathrm{C}$ for $72 \mathrm{~h}$ to promote parasite release from the PMN. Viable parasites were estimated using a Neubauer-counting chamber after $24 \mathrm{~h}$ and $72 \mathrm{~h}$ of incubation.

\subsection{Statistical analysis}

The non-parametric Wilcoxon Signed Ranks Test was used to compare variables of two dependent samples from resting-PMN and $L$. infantum-exposed PMN and, viability and replication levels of parasites that have contacted PMN and cultured parasites (that have not contacted PMN) in relation to all parameters studied and time points. Results are represented as median, percentiles, maximum and minimum values or by mean values \pm standard error, according to the experimental procedures. Differences were considered significant with a $5 \%$ significance level $(p<0.05)$. Statistical analysis was performed with the SPSS 2.0 for Windows software (SPSS Inc., USA) using samples of 10 dogs evaluated in triplicate or values of three independent experiments.

\section{Results}

\subsection{Dog PMN efficiently bind L. infantum promastigotes}

Resting-PMN cultures were used to identify the $\mathrm{GFP}^{-}$cells (Fig. 1A). After $1.5 \mathrm{~h}$ of incubation, the percentage of PMN associated with the parasite $\left(\mathrm{GFP}^{+}\right.$cells, Fig. 1B) was $33.7 \%(19.43 \%, 44.53 \%)$ [median (interquartile range)]. After $3 \mathrm{~h}\left(\mathrm{GFP}^{+}\right.$cells, Fig. 1C), the amount of PMN bound to $L$. infantum-GFP slightly decreased to $28.75 \%$ $(20.83 \%, 37.85 \%)$ and after $4.5 \mathrm{~h}_{\left(\mathrm{GFP}^{+}\right.}$cells, Fig. 1D) about $29.0 \%$ (21.10\%, 36.90\%) of PMN still were associated with parasites (Fig. 1E). However, the amount of PMN associated parasites did not present significant oscilations during the incubation period. Furthermore, the level of GFP fluorescence displayed by the subpopulation $\mathrm{GFP}^{+} \mathrm{PMN}$, 

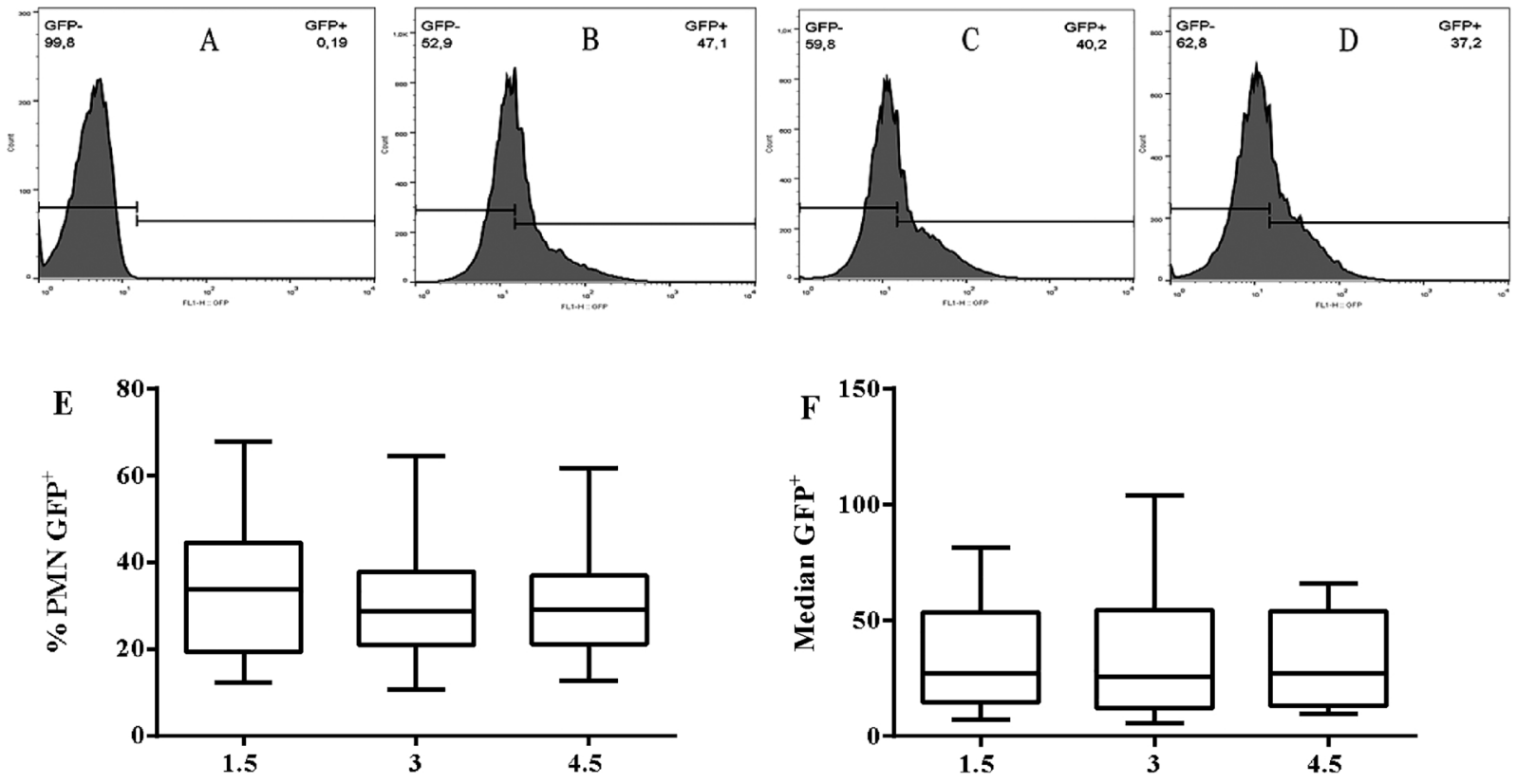

Time (h)

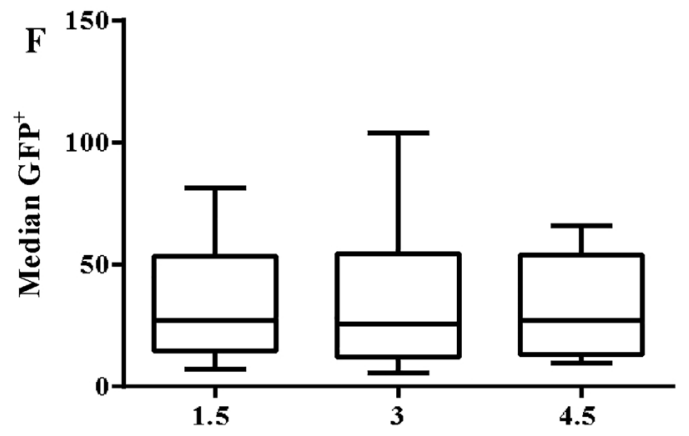

Time (h)

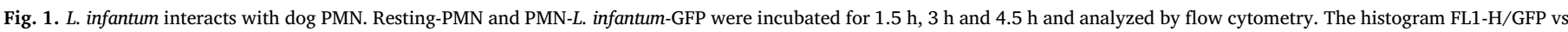

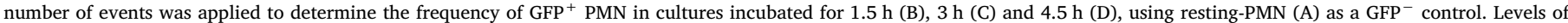

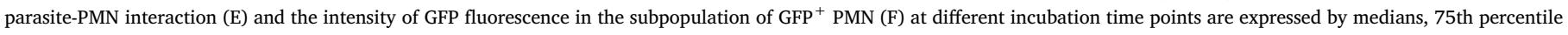
and 25th percentile, and whiskers representing the highest and lowest values.

expressed as the GFP median, remained unchanged throughout the incubation period (Fig. 1F). Thus, the last time point ( $4.5 \mathrm{~h}$ ) was abandoned, because it did not contribute to increase the percentage of infected PMN and was associated with a boost of cell death.

\subsection{L. infantum promastigotes bind to dog PMN in an orientated manner and are engulfed via funnel-like pseudopods}

Parasites adhesion to dog PMN through the aflagellar pole (Fig. 2A and $\mathrm{B}$ ) was uncommon, reaching only $16.7 \%$ of the interactions. The binding through the flagellum tip (Fig. 2C-F) was observed in $63.9 \%$ of the interactions and by another parasite body site in $19.4 \%$ (Fig. $2 \mathrm{G}$ and
$\mathrm{H})$ of the cases. The engulfment of promastigotes takes place via funnellike extensions of the phagocyte surface over the parasite (Fig. 2A-C).

\subsection{Dog neutrophils efficiently internalize L. infantum promastigotes}

The promastigote was the most frequent form observed inside PMN (Fig. 3A and B), but in some cells the parasite was found in the amastigote-like form (Fig. 3C-E). A high proportion of PMN internalized two (Fig. 3B and C), three (Fig. 3D), four (Fig. 3E) or more parasites. In a few dogs's samples, the parasite was detected almost exclusively outside the cell, closely associated with PMN membrane, forming concentric layers (Fig. $3 \mathrm{~F}$ and $\mathrm{G}$ ). After $1.5 \mathrm{~h}$ of incubation only $6 \%(5 \%$,

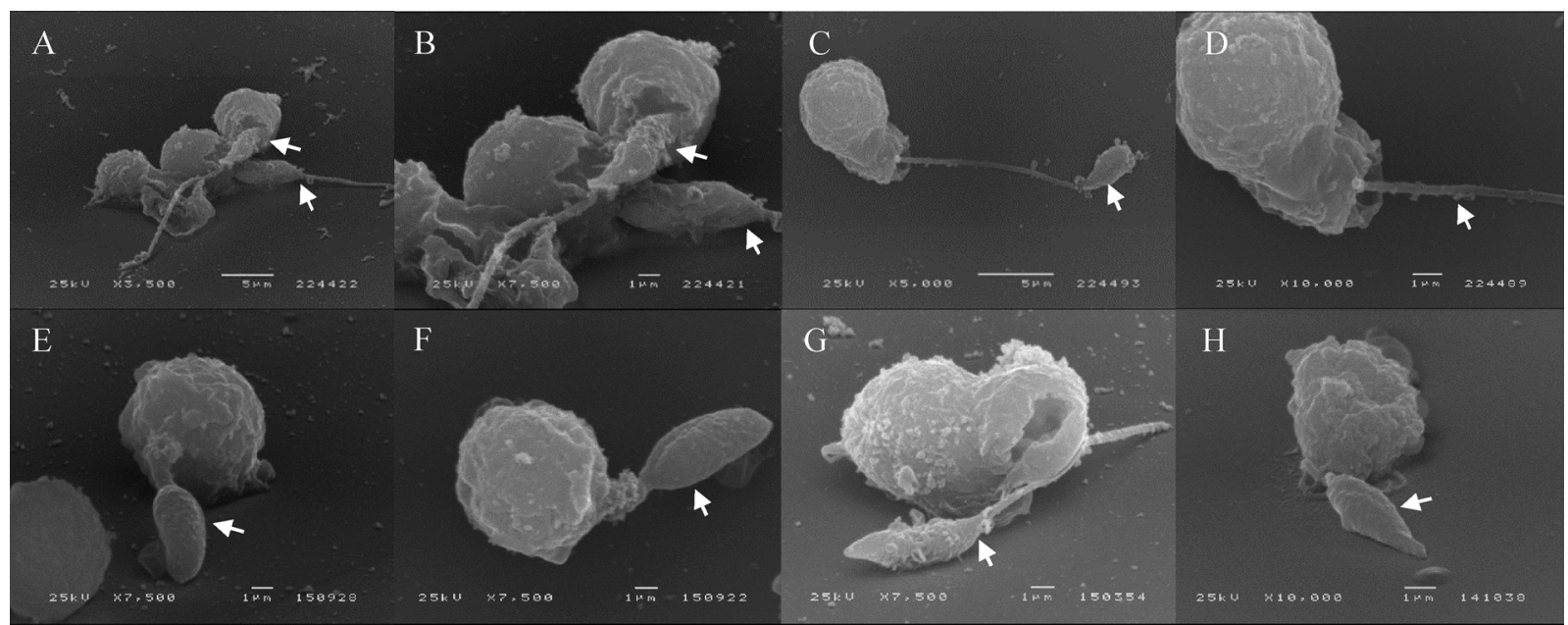

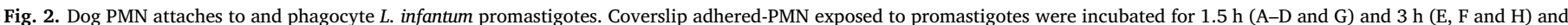

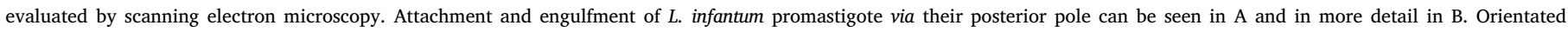

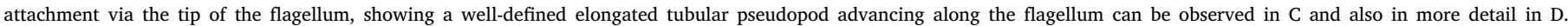

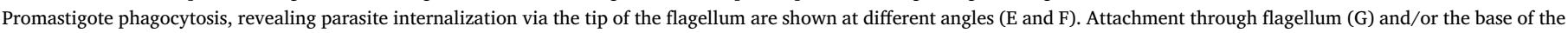
flagellum $(\mathrm{H})$ can be also seen. White arrow - promastigote. 

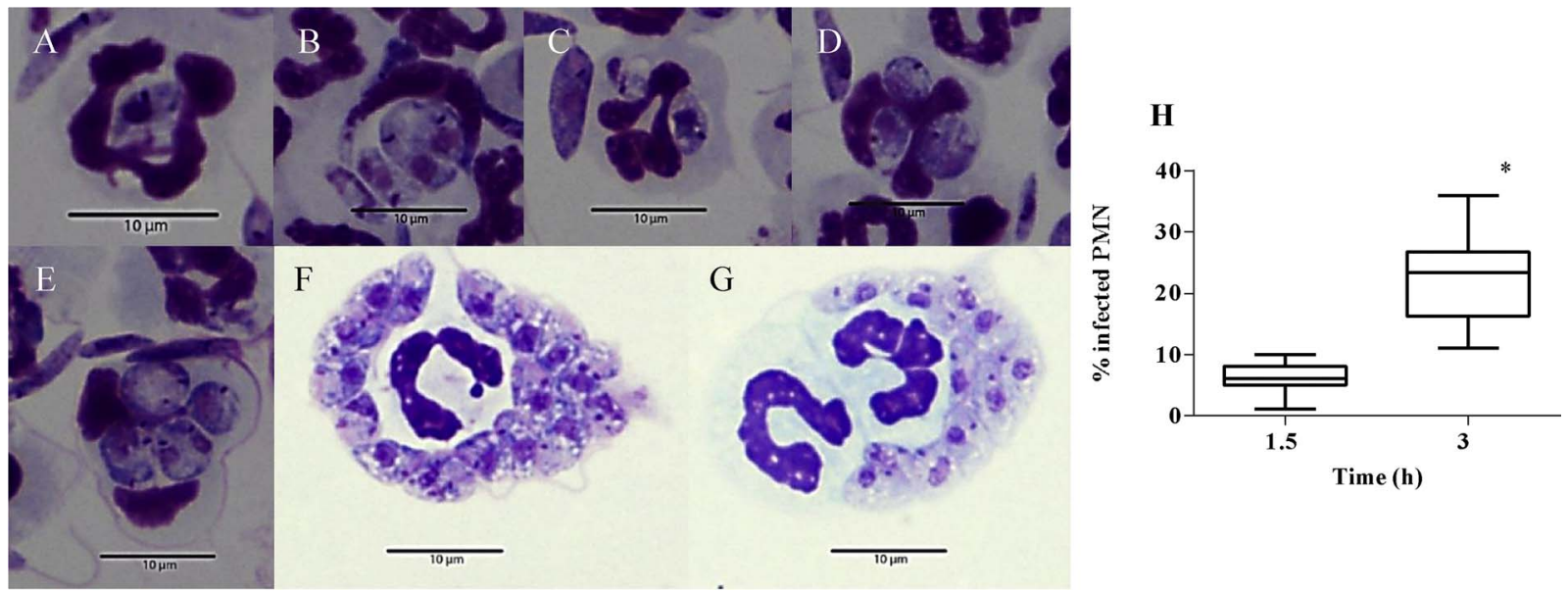

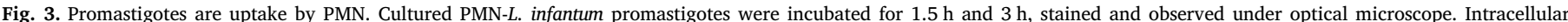

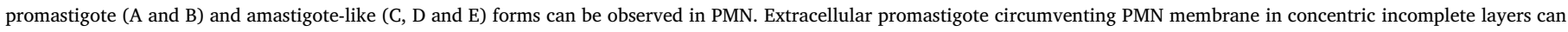

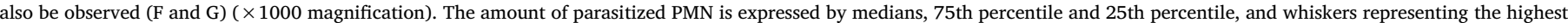

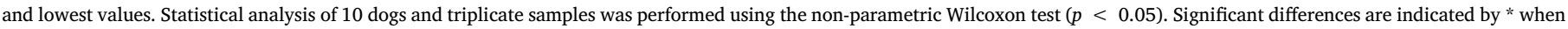
comparing $1.5 \mathrm{~h}$ vs $3 \mathrm{~h}(\mathrm{H})$.

$8 \%$ ) of PMN were parasitized, but later on $23.5 \%$ (16.25\%, 26.75\%) of cells internalized the parasite, showing a significant increase $(p<0.001)$ (Fig. 3H).

\subsection{Previous contact with $L$. infantum decreases PMN migration}

Resting-PMN migration was significantly higher towards viable Leishmania promastigotes $(p=0.001)$ and LCF $(p=0.003)$ when compared stimuli absence (medium). Interestingly, the migration of PMN previously exposed to $L$. infantum promastigotes decreased significantly toward medium $(p=0.008)$, Leishmania promastigotes $(p=0.003)$ and LCF $(p=0.002)$ compared with resting-PMN in equivalent conditions (Fig. 4).

\subsection{L. infantum stimulates dog PMN oxidative burst}

The intracellular production of $\mathrm{O}_{2}{ }^{-}$by PMA-stimulated PMN $(p<0.001)$, L. infantum-exposed PMN $(p<0.001)$ and PMA-stimulated PMN exposed to L. infantum ( $p<0.001$ ) was significantly higher compared with resting-PMN at both $1.5 \mathrm{~h}$ (Fig. 5A) and $3 \mathrm{~h}$ (Fig. 5B) of incubation. As expected, double-stimulated PMN (by PMA and L. infantum) for $1.5 \mathrm{~h}$ produced significantly higher amounts of $\mathrm{O}_{2}{ }^{-}$compared with PMA-stimulated PMN $(p<0.001)$. However, double-

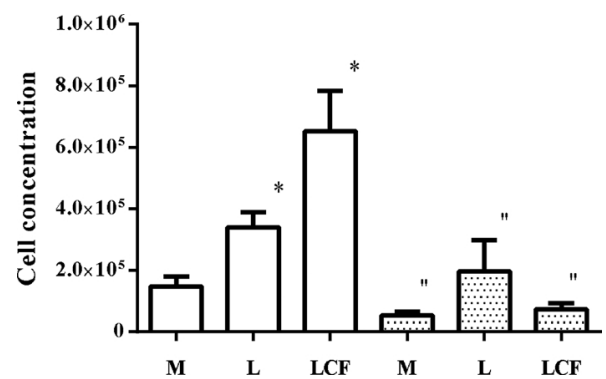

Fig. 4. $L$. infantum induce chemotaxis of dog PMN. The amount of resting-PMN (white bars) and PMN previously exposed to L. infantum promastigotes (dot bars) that migrate from the upper to the lower compartment of a modified Boyden chamber filled with culture medium (M), alive Leishmania promastigotes (L), and Leishmania chemotactic factor (LCF) are indicated. Results of triplicate samples of 10 dogs are presented as mean \pm standard error. Statistical analysis was performed using the Wilcoxon test $(p<0.05)$. Significant differences are represented by * when comparing M (negative control) vs the other conditions, and by " when comparing resting-PMN $v s \mathrm{PMN}+\mathrm{L}$ in equivalent conditions. stimulated PMN exhibited significantly lower production of $\mathrm{O}_{2}{ }^{-}$at $3 \mathrm{~h}$ compared with $1.5 \mathrm{~h}(p=0.013)$.

\subsection{L. infantum regulates neutrophil elastase (NE) exocytosis}

NE activity was significantly higher in supernatants of PMN-L. infantum cultures incubated for $1.5 \mathrm{~h}(p<0.001)$ (Fig. 6A) and $3 \mathrm{~h}\left(p_{0}\right.$ $\min =0.001, p_{15} \min , 30 \mathrm{~min}<0.001$ ) (Fig. 6B), and in supernatants of LPS-stimulated PMN for $1.5 \mathrm{~h}\left(p_{0} \min , 15 \mathrm{~min}=0.012, p_{30 \mathrm{~min}}=0.025\right)$ compared with resting-PMN. Furthermore, at $1.5 \mathrm{~h}$ the release of NE by L. infantum-exposed PMN was higher than the positive control $(p=0.012)$. However, NE exocytosis by PMN exposed to $L$. infantum for $3 \mathrm{~h}$ was lower compared with $1.5 \mathrm{~h}$ cultures $\left(p_{0} \min =0.015\right)$.

\subsection{L. infantum precludes NET formation}

NET were observed as thin filamentous structures or as large cloudlike formations. As expected resting-PMN did not release NET (Fig. 7A and G). $L$. infantum induced a lower NET emission at both time points (1.5 h and $3 \mathrm{~h}$ ) (Fig. 7B and G). However, NET emission by PMA-stimulated PMN was intense (Fig. 7C and G) and PMA-stimulated cells exposed to promastigotes also showed an exuberant NET release (Fig. 7D-G). A close contact between NET and the parasite was observed in PMA-stimulated PMN exposed to $L$. infantum promastigotes (Fig. 8).

Immunolabeling assays showed extracellular structures stained with DAPI and histone H1-FITC, indicating the emission of nuclear DNA and of nuclear proteins. The parasite induced NET release, but to a lesser extent than PMA-stimulated and PMA-stimulated PMN exposed to $L$. infantum. As expected, resting-PMN did not show histone or DNA release (Fig. 9).

\subsection{L. infantum impacts PMN cell death}

Campto stimulation $\left(p_{1.5 \mathrm{~h}}=0.011, p_{3 \mathrm{~h}}=0.008\right)$ and L. infantum exposure $\left(p_{1.5} \mathrm{~h}\right.$ and $\left.3 \mathrm{~h}<0.001\right)$ had a negative impact on PMN viability at both time points $(1.5 \mathrm{~h}$ and $3 \mathrm{~h})$ compared with resting-PMN. The proportion of viable cells was significantly higher in Leishmaniaexposed PMN compared with Campto-stimulated PMN at $1.5 \mathrm{~h}$ $(p=0.008)$, but later on $(3 \mathrm{~h})$ the situation was reversed $(p=0.038)$ (Fig. 10A). Campto (apoptosis inducer) caused a significant increase in PMN apoptosis compared with resting-PMN and parasite-exposed PMN $(p=0.008)$, but only in late cultures ( $3 \mathrm{~h}$ ) (Fig. 10B). The proportion of 

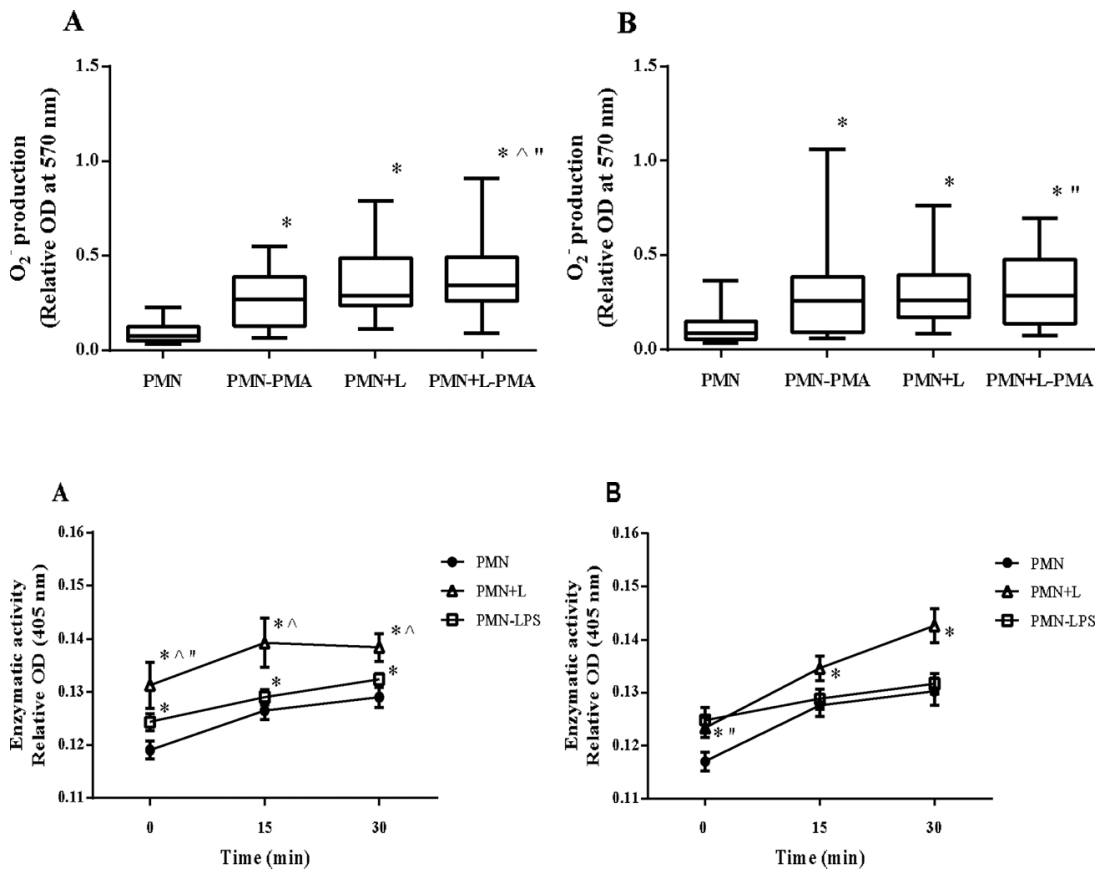

Fig. 6. Enzymatic activity of PMN primary granules. Neutrophilic elastase (NE) exocytosis was quantified in supernatants of PMN, $L$. infantum-exposed PMN (PMN $+\mathrm{L}$ ) and PMN stimulated by LPS (PMNLPS) for $1.5 \mathrm{~h}(\mathrm{~A})$ and $3 \mathrm{~h}$ (B). Supernatants were incubated for $0 \mathrm{~min}$, $15 \mathrm{~min}$ and $30 \mathrm{~min}$ with the specific colorimetric substrate and read at $405 \mathrm{~nm}$. Results of triplicate samples of 10 dogs are presented as mean \pm standard error. Statistical analysis was performed using the Wilcoxon test $(p<0.05)$. Significant differences are indicated by * when comparing PMN (negative control) vs the different conditions, ^ when comparing PMN-LPS vs PMN $+\mathrm{L}$ and by " when comparing $\mathrm{PMN}+\mathrm{L}$ at $1.5 \mathrm{~h}$ vs $3 \mathrm{~h}$.
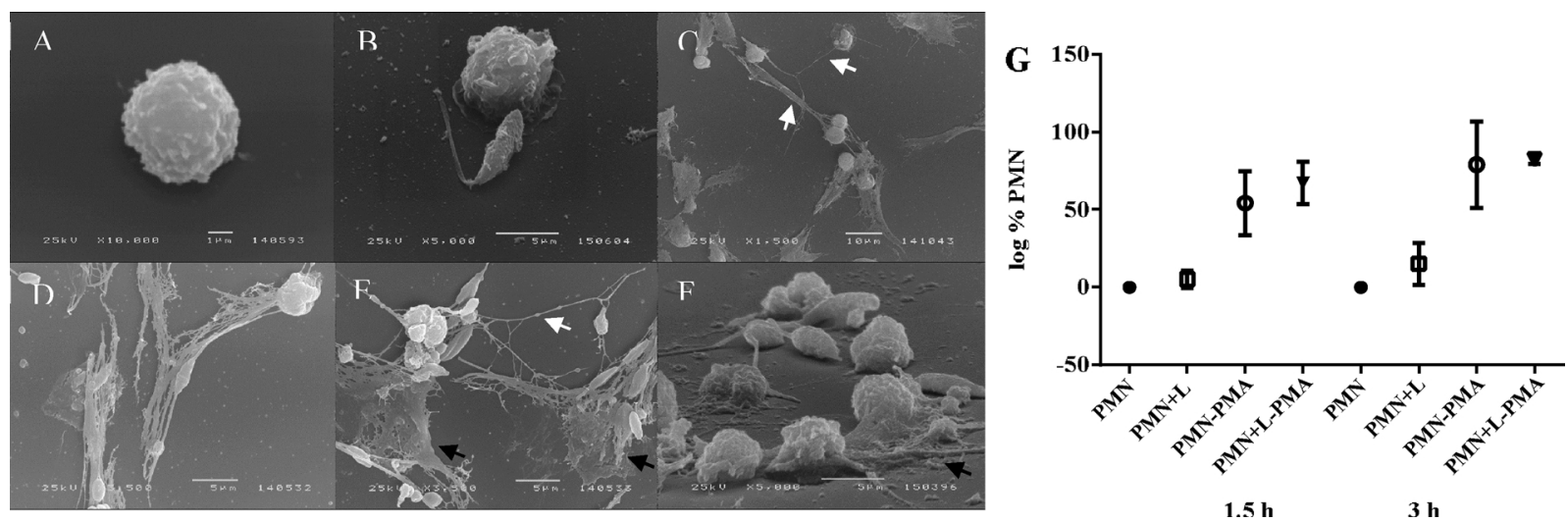

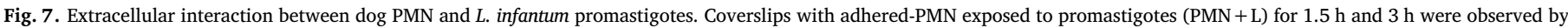

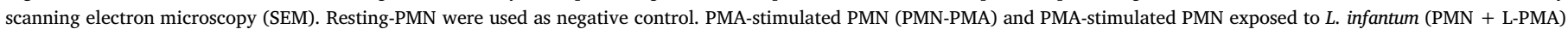

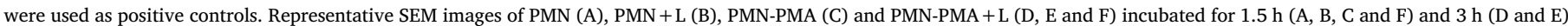

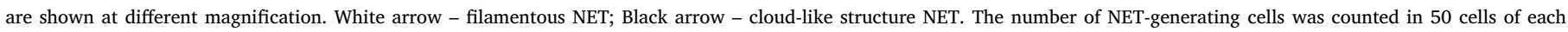
condition and the results are represented by the mean \pm standard deviation of triplicates of three independent experiments (G).

total necrotic cells presented important increases in parasite-exposed PMN ( $p_{1.5} \mathrm{~h}$ and $\left.3 \mathrm{~h}<0.001\right)$ and Campto-stimulated PMN $\left(p_{1.5}\right.$ $\left.\mathrm{h}=0.005, p_{3 \mathrm{~h}}=0.008\right)$ compared with resting-PMN at both time points. Furthermore, a higher time of contact $(3 \mathrm{~h})$ with the parasite increased the proportion of total necrotic cells compared with Camptostimulation ( $p=0.021)$ (Fig. 10C). Interestingly, secondary necrotic cells prevailed at all time points, regarding the variable conditions [resting-PMN $\left(p_{1.5} \mathrm{~h}<0.001\right)$, parasite-exposed PMN $\left(p_{1.5} \mathrm{~h}\right.$ and 3 $\mathrm{h}<0.001)$ and Campto-stimulated PMN $\left(p_{1.5} \mathrm{~h}\right.$ and $\left.\left.3 \mathrm{~h}=0.008\right)\right]$, except in resting-PMN incubated for $3 \mathrm{~h}$.

The proportion of primary and also secondary necrotic cells in parasite-exposed PMN $\quad\left(p_{1.5} \quad \mathrm{~h}<0.001, \quad p_{3 \mathrm{~h}}=0.005\right.$ and $p_{1.5 h a n d 3 h}<0.001$, respectively) and Campto-stimulated PMN ( $p$ $1.5 \mathrm{~h}=0.001, p_{3 \mathrm{~h}}=0.028$ and $p_{1.5 \mathrm{hand} 3 \mathrm{~h}}=0.008$, respectively) was significantly higher in comparison with resting-PMN at both time points. While the proportion of primary necrotic cells was higher in Campto-stimulated PMN compared to parasite-exposed cultures $(p<0.001)$ at $1.5 \mathrm{~h}$, the amount of secondary necrotic cells was superior in parasite-exposed cultures compared with Campto-stimulated
PMN at both time points $\left(p_{1.5 \mathrm{hand} 3 \mathrm{~h}}=0.038\right)($ Fig. 10D).

\subsection{L. infantum delays apoptosis of parasitized neutrophils}

Microscopic observation of $L$. infantum-exposed PMN and of restingPMN revealed the presence of apoptotic cells exhibiting the classical features, such as cell rounding and shrinking, and chromatin condensation (pycnotic nuclei), which tends to marginate, acquiring a crescent form (Fig. 11A and B). Late stages of PMN apoptosis were also observed as nuclear fragmentation (karyorrhexis, apoptotic bodies) (Fig. 11B and C). Although some infected PMN exhibited apoptotic nuclear morphology, after $3 \mathrm{~h}$ of parasite exposure most of the apoptotic PMN were not parasitized.

3.10. PMN reduce L. infantum promastigote viability by extracellular and intracellular parasite killing

L. infantum parasites previously exposed to PMN intracellular and extracellular killing mechanisms showed reduced viability when 


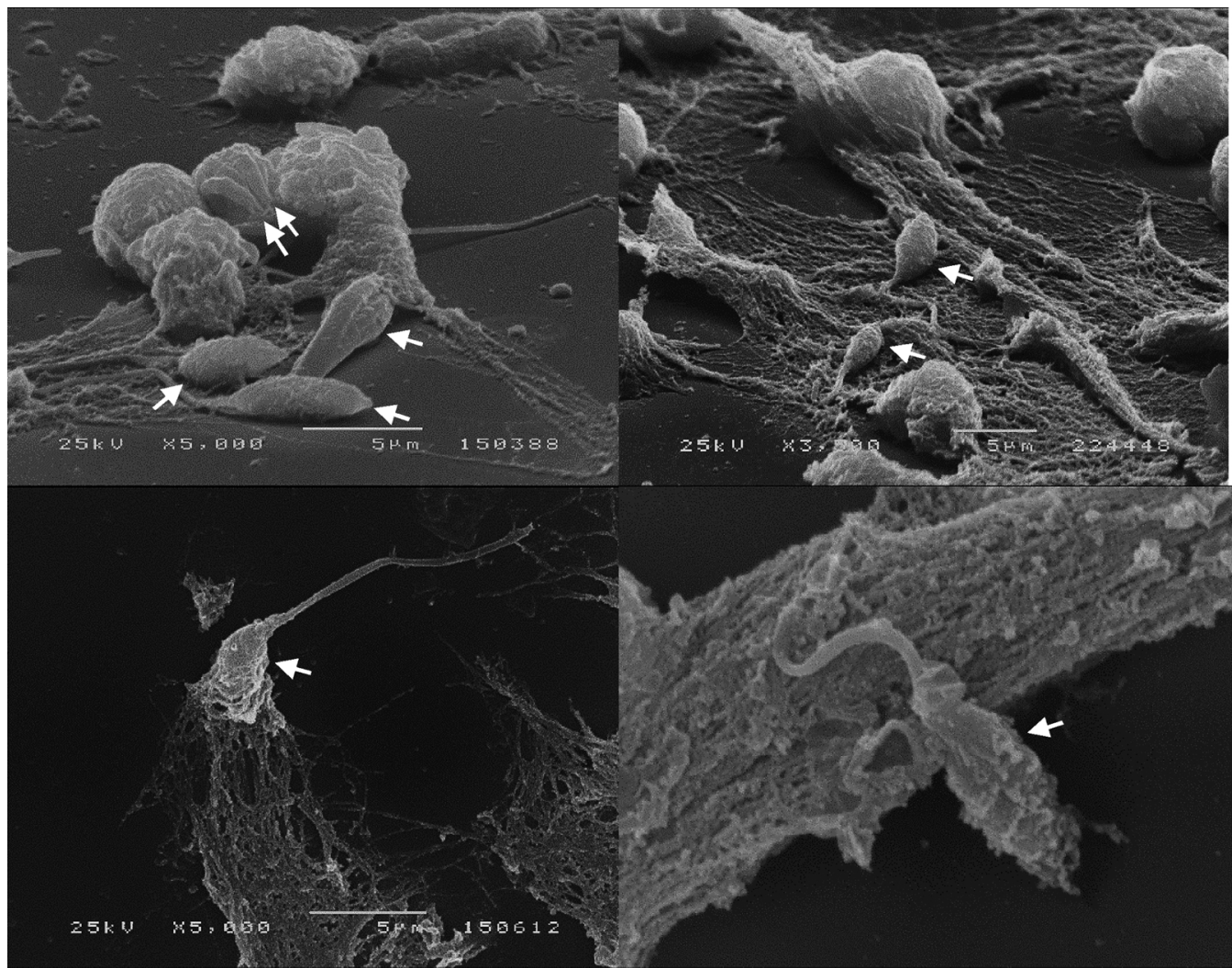

Fig. 8. Neutrophil extracellular traps limit parasite spread. Coverslips with adheredPMN stimulated with PMA and exposed to $L$. infantum promastigotes for $3 \mathrm{~h}$ were observed by scanning electron microscopy. A close interaction between NET and the parasite (white arrows) evidence an entrapping effect.

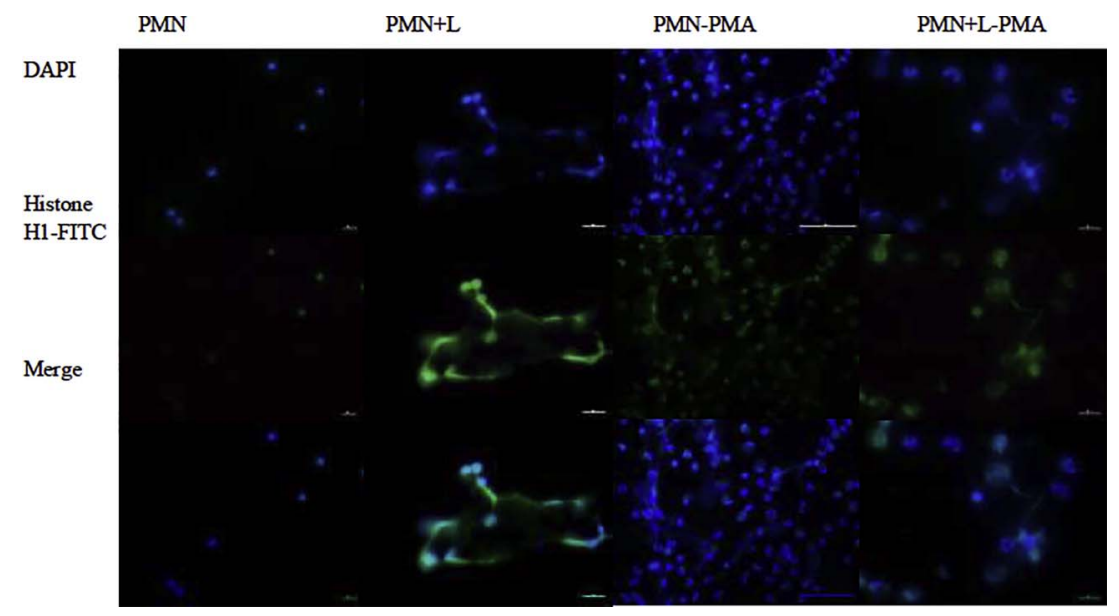

Fig. 9. NET release evaluated by histone immunolabeling. Coverslips with adhered-PMN exposed to promastigotes $(\mathrm{PMN}+\mathrm{L})$ for $3 \mathrm{~h}$ were prepared for immunolabeling. Additional resting-PMN (PMN, negative control), PMN stimulated with PMA (PMN-PMA) (positive control) and PMN stimulated with PMA and exposed to L. infantum (PMN + L-PMA) were stained with anti-histone H1 FITC and DAPI. Representative images of cells stained with DAPI and anti-histone H1 and the respective merge images $(\times 600$ or $\times 1000$ magnification) show the extracellular release of histone.

compared with $L$. infantum promastigote cultures $(p<0.001)$ (Fig. 12). Optical microscopy revealed that some intracellular parasites preserved the structural integrity. However, images of PMN containing large vacuoles surrounding cellular debris that distort cellular morphology suggest parasite death (Fig. 13). Evidences of degraded parasites were more frequent in some particular dogs.

Some TEM images showed PMN interacting with extracellular parasites that have lost their normal ultrastructure: nucleus appeared more electro-dense and the presence of cytoplasmic blebs denotes loss of membrane integrity (Fig. 14A-D). Intracellular degraded parasites were observed within spacious phagosomes (Fig. 14E and F). Occasionally, the advanced intracellular degradation process prevented the identification of any parasitic organelle.
3.11. L. infantum maintains the viability and the capacity of multiplication after neutrophil phagocytosis

In order to assess the viability of internalized parasites, PMN incubated with $L$. infantum were subjected to external parasite removal. After incubation, $5 \times 10^{5}\left(3.3 \times 10^{5}, 8.3 \times 10^{5}\right)$ moving parasites were counted, suggesting that the internalized parasites actively escape from PMN or were released after PMN death, maintaining the viability. Furthermore, after $72 \mathrm{~h}$ of incubation a significantly higher $(p<0.001)$ number of viable parasites were found) (Fig. 15).

\section{Discussion}

The interaction between canine PMN and L. infantum is largely unknown. In a previous in vivo study our group demonstrated that the 

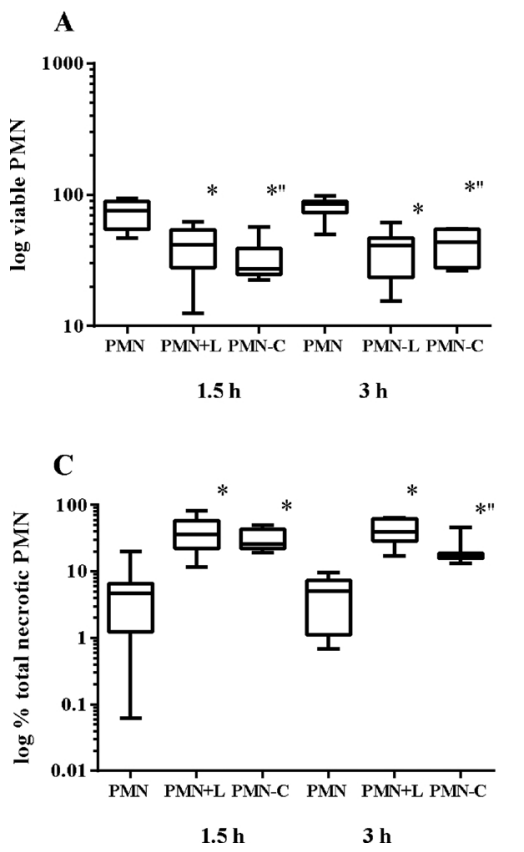

\section{B}
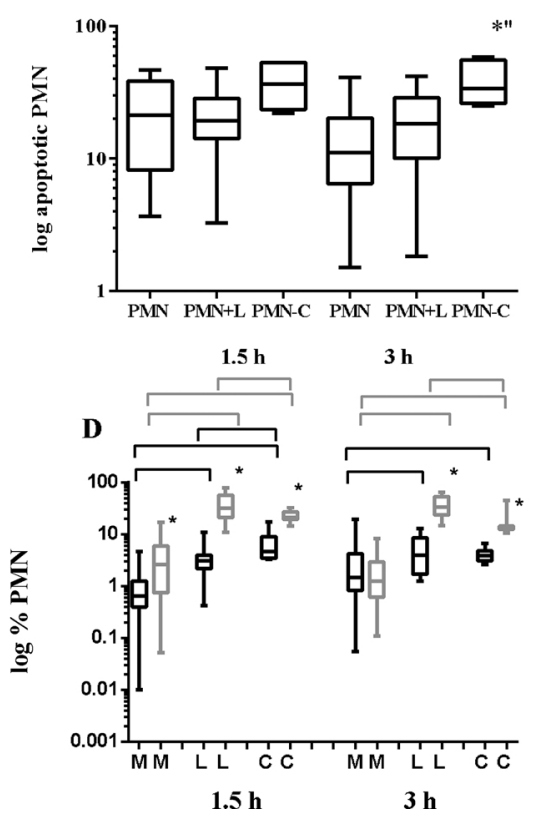

Fig. 10. Levels of viable, apoptotic and necrotic dog PMN after exposure to $L$. infantum promastigotes. PMN exposed to $L$. infantum $(\mathrm{PMN}+\mathrm{L})$ were treated with annexin V FITC and propidium iodine (PI) and analyzed by flow cytometry. Resting-PMN and (S)(+)-camptothecin-stimulated PMN (PMNC) were used as negative and apoptotic positive controls, respectively. Frequency of viable (A), apoptotic (B), total necrotic (C) and of primary and secondary necrotic cells (D) of triplicate samples of 10 dogs is presented by medians, 75 th percentile and 25 th percentile and, whiskers representing the highest and lowest values. Statistical analysis was performed using the Wilcoxon test $(p<0.05)$. Significant differences are represented by * when comparing PMN (negative control) vs the other conditions, " when comparing $\mathrm{PMN}+\mathrm{L}$ vs $\mathrm{PMN}+\mathrm{C}(\mathrm{A}-\mathrm{C})$ and when comparing primary vs secondary necrotic cells in the same condition. Black and gray connecting lines indicate significant differences in primary and secondary necrotic cells, respectively (D).

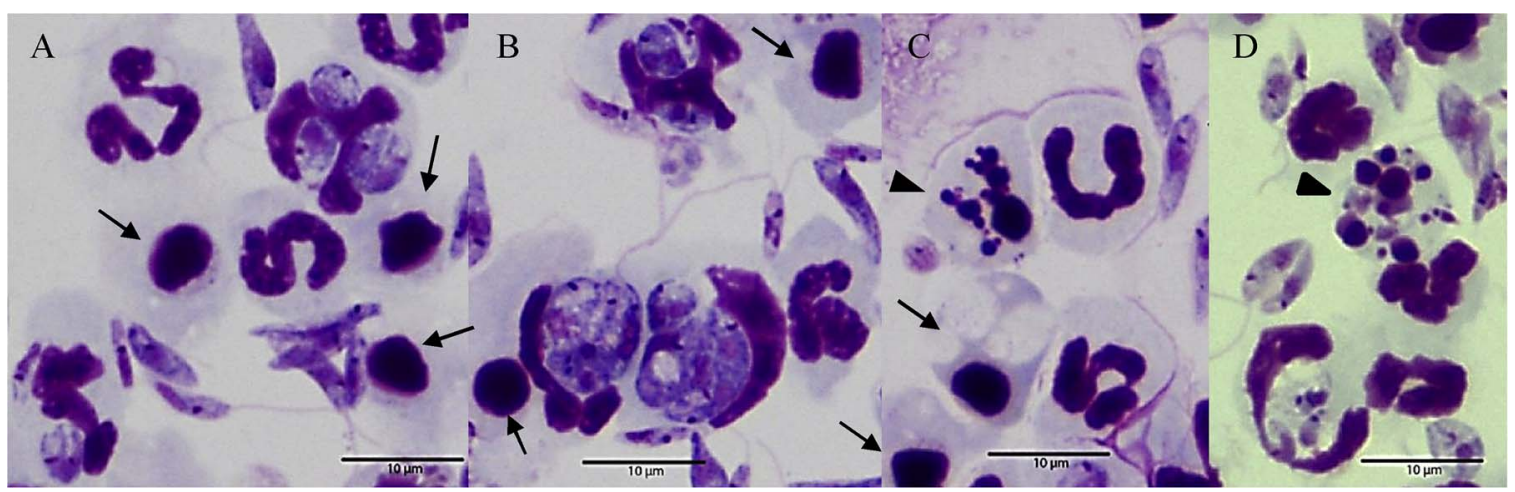

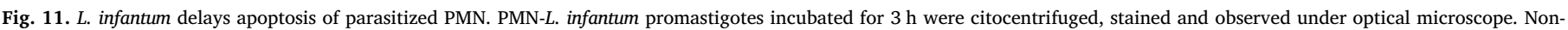
parasitized early (black arrow) (A, B, C) and late (arrow head) apoptotic cells (C, D) can be observed ( $\times 1000$ magnification).

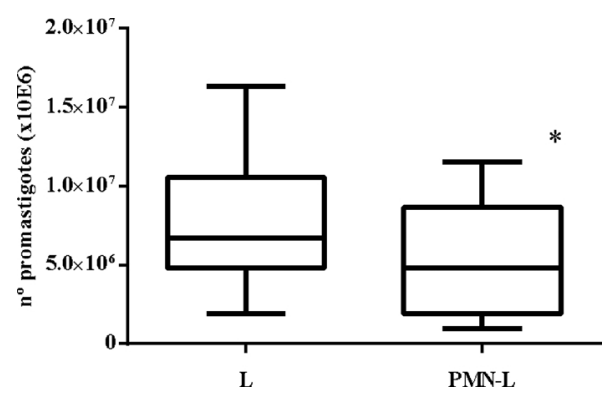

Fig. 12. Exposure to dog PMN has a negative impact in $L$. infantum survival. After incubation with PMN for $24 \mathrm{~h}(\mathrm{PMN}+\mathrm{L})$ active $L$. infantum promastigotes were quantified in a Neubauer-counting chamber. Promastigotes not exposed to PMN (L) were used as positive control. The amount of viable parasites is express by medians, 75th percentile and 25th percentile and, whiskers representing the highest and lowest values. Statistical analysis of triplicate samples of 10 dogs was performed using the Wilcoxon test $(p<0.05)$. Significant differences are represented by * when comparing L vs PMN $+\mathrm{L}$.

experimental injection of dogs with $L$. infantum promastigotes promotes a rapid dermal infiltration of PMN (Santos-Gomes et al., 2000). In the present study it becomes clear that live $L$. infantum promastigotes and culture supernatants induce a strong PMN migration, suggesting that some excretory/secretory substances released by the parasite are chemoattractant for PMN. Thus, Leishmania modulates leukocyte recruitment at the early phase of infection, favoring its internalization and consequently protection against soluble innate immune components, such as complement components that can destroy extracellular parasites. However, the previous contact with $L$. infantum inhibited PMN migration, which can promote the retention of infected cells at the inoculation site, ensuring parasite protection until macrophage arrival and its subsequent transference to the definitive host cell.

Although promastigotes rapidly adhere to PMN surface, this is not a random interaction. Promastigotes preferentially adhere to PMN by the flagellum tip, possibly reflecting the main concentration of adhesion molecules (adhesiotopes) (Rittig and Bogdan, 2000). The attachment of L. infantum via the flagellum tip seems to promote the extend of symmetrical pseudopods, that maintain the directional entry of the parasite into PMN (symmetrical phagocytosis). The type of phagocytosis seems to influence parasite fate with symmetrical phagocytosis favoring parasite killing (Hsiao et al., 2011).

Although a relative high proportion of PMN rapidly interacts with the parasite, promastigote internalization requires a longer contact time. Moreover, in some dogs the parasite was detected almost exclusively outside the cell. Taking into account that parasite undergoes phagocytosis as a passive partner, these findings point towards different PMN responses to $L$. infantum in a host dependent manner. Dog genetic background, breed, age, sex and lifestyle may account for the different 


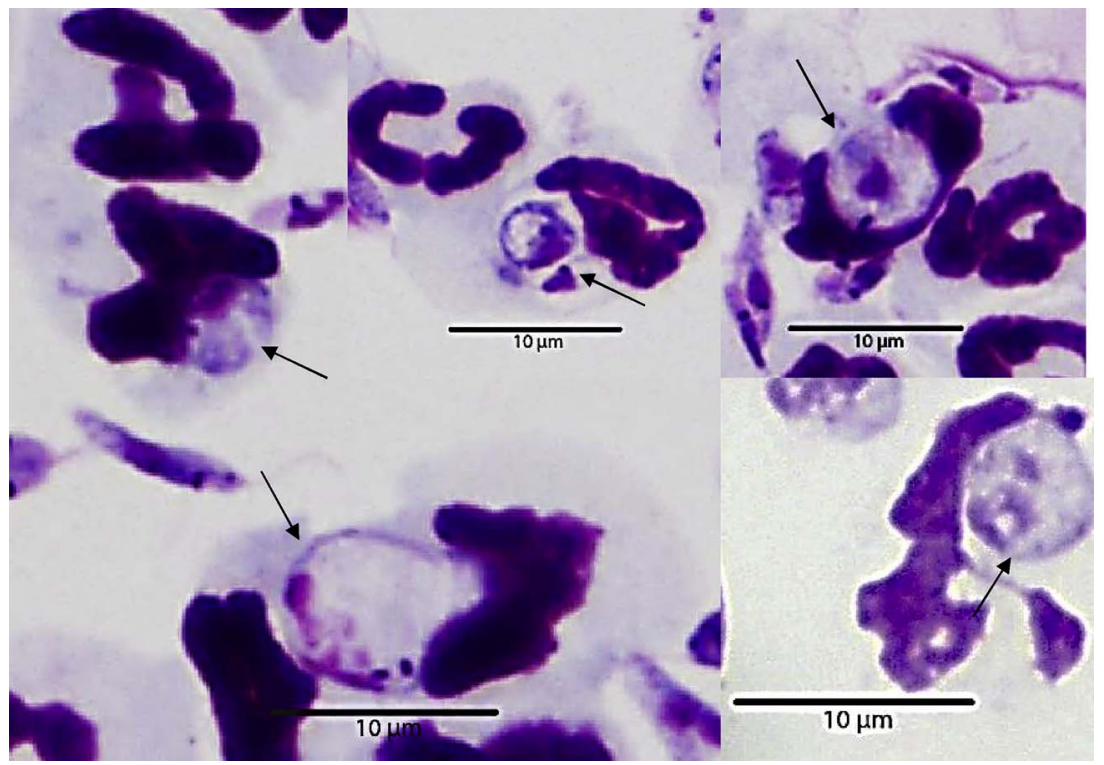

Fig. 13. Large phagolysosomes ensure parasite killing. PMN exposed to $L$. infantum promastigotes for $3 \mathrm{~h}$ were citocentrifuged, stained and observed under optical microscope. Parasites that lost the structural integrity can be found inside large phagosomes (arrow) $(\times 1000$ magnification)

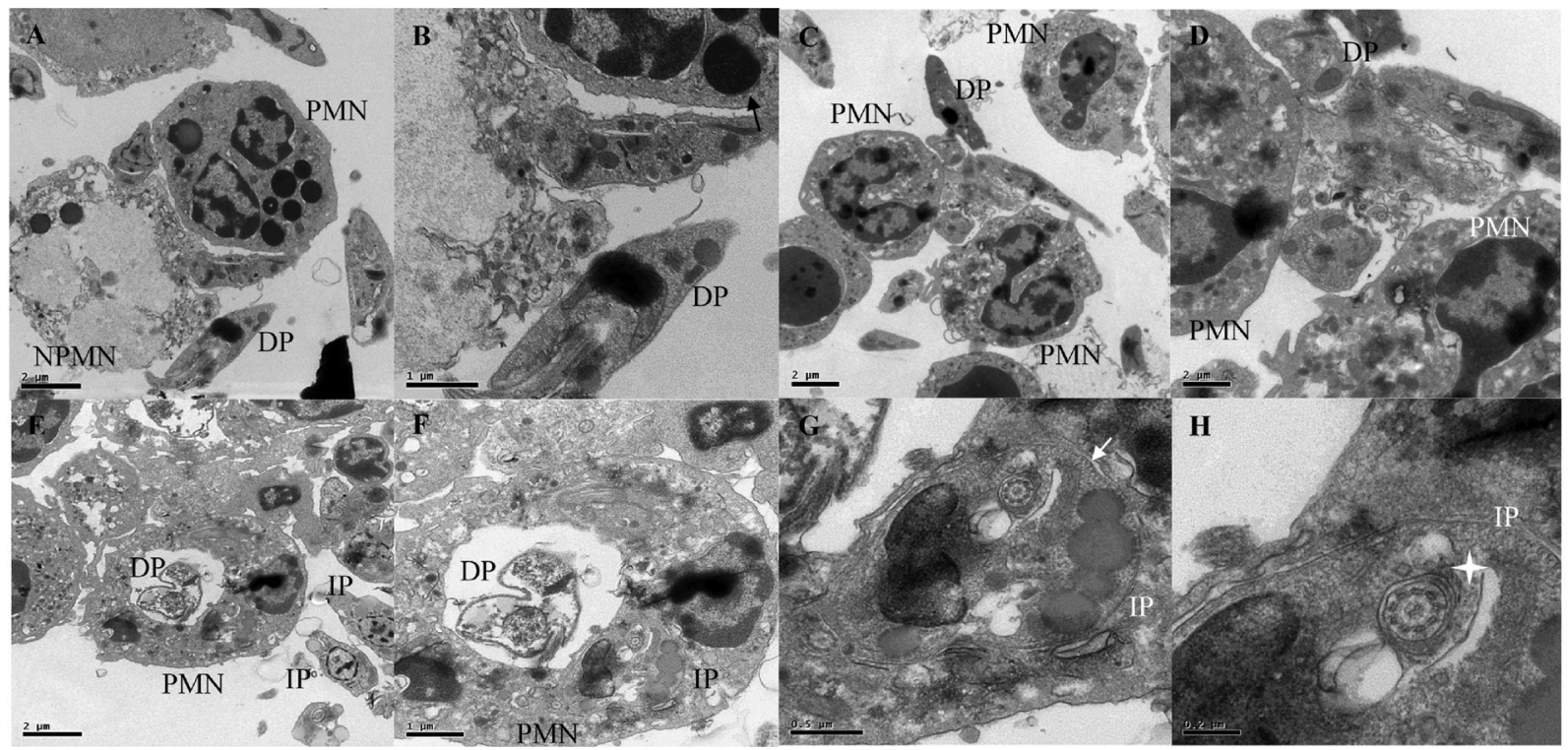

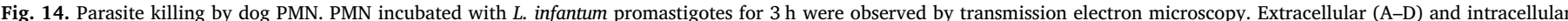

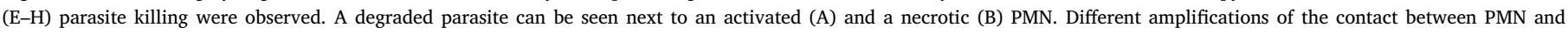

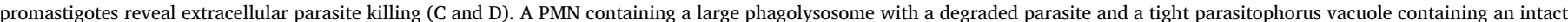



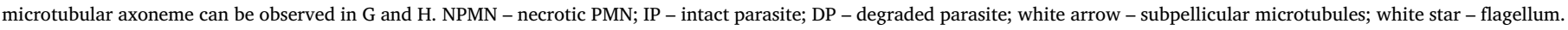

phagocytic capacity of PMN.

The majority of parasites internalized by dog PMN keeps an elongated appearance, suggesting that promastigotes did not differentiate into the amastigote form as observed by other authors (van Zandbergen et al., 2004). Probably, intracellular environment of PMN does not provide the required conditions for the differentiation process and/or the time that the parasite remains inside PMN is not sufficient to complete this process. This observation reinforces the role of PMN as ordinary transitional host cells.

Parasite phagocytosis induced a sudden and strong oxidative burst that with time turns out to be suppressed, probably due to PMN death or parasite derivative ROS-deactivating molecules (Longoni et al., 2013; Kima, 2014). $\mathrm{O}_{2}{ }^{-}$production may have a negative impact on $L$. infantum survival, as already described in $L$. donovani and $L$. major promastigotes (Pearson and Steigbigel, 1981; Laufs et al., 2002). Indeed, internalized parasites showing no structural integrity were found inside large vacuoles, pointing towards phagocytosis dependent killing. However, this kind of images were more frequent in some dogs, indicating different responses depending on the cell donor. Similar to previously described by Gueirard et al. (2008), two types of phagosomes were found in the same PMN: large spacious phagosomes containing degraded parasites and tight phagosomes with intact parasites, suggesting that different parasites induce different PMN responses. Promastigotes used in the present study were in the stationary growth phase, which comprise a heterogeneous population of parasites with different abundance of virulent factors, namely gp63 and LPG (Ueno and Wilson, 2012). Thus, it can be hypothesized that non-virulent or less virulent promastigotes can be killed by dog PMN while virulent parasites may subvert the effector machinery of PMN, surviving and giving origin to a Leishmania infection in the dog.

Extracellular effector mechanisms, such as NET release and enzyme exocytosis also can contribute to parasite killing. Although it is already 


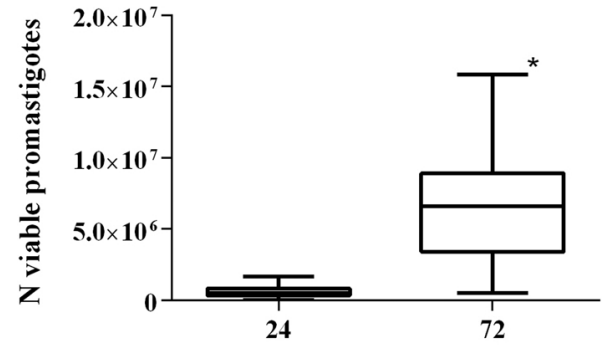

Time (h)

Fig. 15. PMN intracellular parasites are viable and retain replication capability. After removing the extracellular parasites, PMN-L. infantum promastigotes incubated for $3 \mathrm{~h}$ were transferred to Schneider medium and incubated at $24^{\circ} \mathrm{C}$. Viable promastigotes estimated at $24 \mathrm{~h}$ and $72 \mathrm{~h}$ are represented by medians, 75th percentile and 25th percentile, and whiskers indicating the highest and lowest values. Statistical analysis of triplicate samples of 10 dogs was performed using the Wilcoxon test $(p<0.05)$. Significant differences are represented by * when comparing $24 \mathrm{~h}$ vs $72 \mathrm{~h}$.

described that several Leishmania species induce human PMN to emit NET (es-Costa et al., 2009, 2014; es-Costa et al., 2009, 2014; Gabriel et al., 2010), the present study demonstrates that $L$. infantum almost abolishes NET formation by dog PMN, indicating that the parasite negatively modulate this effector mechanism, favoring parasite spreading and survival, and preventing the detrimental NET-induced pro-inflammatory response. Furthermore, it is reasonable to assume that $\mathrm{NE}$ exocytosis at the early phase of infection contribute to parasite extracellular killing. However, enzyme decreased with infection time, pointing toward a late modulation parasite-induced. Indeed, inhibitors of serine peptidases that were identified in $L$. major and $L$. donovani (Eschenlauer et al., 2009; Alam et al., 2015) can play a role in $L$. infantum survival by negatively regulate the extracellular enzymatic activity.

Although dog PMN reduced significantly parasite burden, a considerable proportion of intracellular parasites are viable and can replicate, probably using some of the evasion strategies already described (Laufs et al., 2002; Gueirard et al., 2008). These surviving parasites can be transferred to the definitive host cell and can perpetuate canine infection.

In addition to the deleterious effects caused by PMN on the parasite, PMN viability is also negatively affected by the parasite, promoting secondary necrosis. This kind of death occurs when cells that undergo apoptosis experience subsequent necrosis and can occur after exposition to a strong stimulus (Iba et al., 2013). Secondary necrosis is characterized by the uncontrolled release of toxic components that promote pro-inflammatory responses, leading to tissue damage (Scaffidi et al., 2002) and generating an unfavorable environment for parasite survival. However, the absence of apoptotic cell removal by scavenger's macrophages can lead to cell elimination by necrosis (Silva, 2010) and justify the high levels of secondary necrosis found.

Although it has been shown that some Leishmania were able to modulate PMN apoptosis, prolonging its life span or accelerating its death (Aga et al., 2002; Gueirard et al., 2008; Carlsen et al., 2013; Falcão et al., 2015), in the present study $L$. infantum infection did not increase apoptosis of dog PMN. In fact, the majority of apoptotic cells was not parasitized, suggesting that at early stages of infection intracellular parasites or even their products protect dog PMN, avoinding cell death. The mechanism implicated in this interaction is unknown, but LPG might be involved (Lüder et al., 2001).

However, it is important to take into account that blood PMN may not act in the same way as PMN that have been recruited to the Leishmania inoculation site (Abebe et al., 2012). Furthermore, in vitro studies may not entirely reflect the in vivo conditions, since additional factors are involved as is the case of sand fly saliva and others components of the host immune system.

The present study highlights the effector functions of dog PMN when exposed to $L$. infantum promastigotes, indicating that at least initially PMN promote the reduction of parasite load through intra and extracellular killing mechanisms. However, the inability of PMN to clear the parasite can favors dog infection. Additional studies are required to clarify the diversity of effector functions found between PMN of different donors, correlating PMN phenotype with dog's susceptibility to $L$. infantum infection.

\section{Funding}

This work was supported by the Portuguese Foundation for Science and Technology (FCT) through research grants (PTDC/CVT/113121/ 2009, PTDC/CVT/118566/2010, UID/Multi/04413/2013 and UID/ CVT/00276/2013) and PhD scholarships (SFRH/BD/73386/2010, SFRH/BD/77055/2011 and SFRH/BD/101467/2014).UID/Multi/ 04413/2013.

\section{Conflict of interest}

The authors declare no competing personal or financial interests.

\section{Acknowledgments}

A special acknowledge to Doutor MaríaVictoria Alarcón Sánchez from Centro de Investigaciones Científicas y Tecnológicas de Extremadura (CICYTEX) for her aid with fluorescence image acquisition, to Telmo Nunes from Centro de Microscopia Eletrónica, Faculdade de Ciências, Universidade de Lisboa for his aid with SEM image acquisition, and to Amélia Fernandes for her help in image treatment.

\section{Appendix A. Supplementary data}

Supplementary data associated with this article can be found, in the online version, at http://dx.doi.org/10.1016/j.vetpar.2017.10.008.

\section{References}

Abebe, T., Hailu, A., Woldeyes, M., Mekonen, W., Bilcha, K., Cloke, T., Fry, L., Seich Al Basatena, N.K., Corware, K., Modolell, M., Munder, M., Tacchini-Cottier, F., Müller, I., Kropf, P., 2012. Local increase of arginase activity in lesions of patients with cutaneous leishmaniasis in Ethiopia. PLoS Negl. Trop. Dis. 6, e1684. http://dx.doi.org/ 10.1371/journal.pntd.0001684.

Aga, E., Katschinski, D.M., van Zandbergen, G., Laufs, H., Hansen, B., Müller, K., Solbach, W., Laskay, T., 2002. Inhibition of the spontaneous apoptosis of neutrophil granulocytes by the intracellular parasite Leishmania major. J. Immunol. 169, 898-905.

Alam, M.N., Das, P., De, T., Chakraborti, T., 2015. Identification and characterization of a Leishmania donovani serine protease inhibitor: possible role in regulation of host serine proteases. Life Sci. 144, 218-225.

Beil, W.J., Meinardus-Hager, G., Neugebauer, D.C., Sorg, C., 1992. Differences in the onset of the inflammatory response to cutaneous leishmaniasis in resistant and susceptible mice. J. Leukoc. Biol. 52, 135-142.

Brinkmann, V., Laube, B., Abu Abed, U., Goosmann, C., Zychlinsky, A., 2010. Neutrophil extracellular traps: how to generate and visualize them. J. Vis. Exp. (36), 1724. http://dx.doi.org/10.3791/1724.

Carlsen, E.D., Hay, C., Henard, C.A., Popov, V., Garg, N.J., Soong, L., 2013. Leishmania amazonensis Leishmania amazonensis amastigotes trigger neutrophil activation but resist neutrophil microbicidal mechanisms. Infect. Immun. 81, 3966-3974.

Carlsen, E.D., Liang, Y., Shelite, T.R., Walker, D.H., Melby, P.C., Soong, L., 2015. Permissive and protective roles for neutrophils in leishmaniasis. Clin. Exp. Immunol. $182,109-118$.

Charan, J., Biswas, T., 2013. How to calculate sample size for different study designs in medical research? Indian J. Psychol. Med. 35, 121-126.

Eschenlauer, S.C.P., Faria, M.S., Morrison, L.S., Bland, N., Ribeiro-Gomes, F.L., DosReis, G.A., Coombs, G.H., Lima, A.P.C.A., Mottram, J.C., 2009. Influence of parasite encoded inhibitors of serine peptidases in early infection of macrophages with Leishmania major. Cell. Microbiol. 11, 106-120.

Falcão, S.A., Weinkopff, T., Hurrell, B.P., Celes, F.S., Curvelo, R.P., Prates, D.B., Barral, A., Borges, V.M., Tacchini-Cottier, F., de Oliveira, C.I., 2015. Exposure to Leishmania braziliensistriggers neutrophil activation and apoptosis. PLoS Negl. Trop. Dis. 9 (3), e0003601. http://dx.doi.org/10.1371/journal.pntd.0003601.

Faria, M.S., Reis, F.C., Lima, A.P., 2012. Toll-like receptors in Leishmania infections: guardians or promoters. J. Parasitol. Res. 2012, 930257. http://dx.doi.org/10.1155/ 2012/930257.

Gabriel, C., McMaster, W.R., Girard, D., Descoteaux, A., 2010. Leishmania donovani 
promastigotes evade the antimicrobial activity of neutrophil extracellular traps. J. Immunol. 185, 4319-4327.

Gueirard, P., Laplante, A., Rondeau, C., Milon, G., Desjardins, M., 2008. Trafficking of Leishmania donovani promastigotes in non-lytic compartments in neutrophils enables the subsequent transfer of parasites to macrophages. Cell. Microbiol. 10, 100-111.

Guimarães-Costa, A.B., Nascimento, M.T., Froment, G.S., Soares, R.P., Morgado, F.N., Conceição-Silva, F., Saraiva, E.M., 2009. Leishmania amazonensis promastigotes induce and are killed by neutrophil extracellular traps. Proc. Natl. Acad. Sci. U. S. A. 106, 6748-6753.

Guimarães-Costa, A.B., DeSouza-Vieira, T.S., Paletta-Silva, R., Freitas-Mesquita, A.L., Meyer-Fernandes, J.R., Saraiva, E.M., 2014. 3'-nucleotidase/nuclease activity allows Leishmania parasites to escape killing by neutrophil extracellular traps. Infect. Immun. 82, 1732-1740.

Helhazar, M., Leitão, J., Duarte, A., Tavares, L, da Fonseca, I.P., 2013. Natural infection of synathropic rodent species Mus musculusand Rattus norvegicus by Leishmania infantum in Sesimbra and Sintra-Portugal. Parasit. Vectors 6, 88. http://dx.doi.org/10. 1186/1756-3305-6-88.

Hsiao, C.H., Ueno, N., Shao, J.Q., Schroeder, K.R., Moore, K.C., Donelson, J.E., Wilson, M.E., 2011. The effects of macrophage source on the mechanism of phagocytosis and intracelular survival of Leishmania. Microbes Infect. 13, 1033-1044.

Hurrell, B.P., Schuster, S., Grün, E., Coutaz, M., Williams, R.A., Held, W., Malissen, B. Malissen, M., Yousefi, S., Simon, H.U., Müller, A.J., Tacchini-Cottier, F., 2015. Rapid sequestration of Leishmania mexicana by neutrophils contributes to the development of chronic lesion. PLoS Pathog. 11 (5), e1004929. http://dx.doi.org/10.1371/ journal.ppat.1004929.

Hurrell, B.P., Regli, I.B., Tacchini-Cottier, F., 2016. Different Leishmania species drive distinct neutrophil functions. Trends Parasitol. 32, 392-401.

Iba, T., Hashiguchi, N., Nagaoka, I., Tabe, Y., Murai, M., 2013. Neutrophil cell death inresponse to infection and its relation to coagulation. J. Intens. Care 1 (1), 13. http://dx.doi.org/10.1186/2052-0492-1-13.

Kima, P.E., 2014. Leishmania molecules that mediate intracellular pathogenesis. Microbes Infect. 16, 721-726.

Lüder, C.G., Gross, U., Lopes, M.F., 2001. Intracellular protozoan parasites and apoptosis: diverse strategies to modulate parasite-host interactions. Trends Parasitol. 17, $480-486$

Laufs, H., Müller, K., Fleischer, J., Reiling, N., Jahnke, N., Jensenius, J.C., Solbach, W., Laskay, T., 2002. Intracellular survival of Leishmania major in neutrophil granulocytes after uptake in the absence of heat-labile serum factors. Infect. Immun. 70, 826-835.

Longoni, S.S., Sánchez-Moreno, M., López, J.E., Marín, C., 2013. Leishmania infantum secreted iron superoxide dismutase purification and its application to the diagnosis of canine Leishmaniasis. Comp. Immunol. Microbiol. Infect. Dis. 36, 499-506.

Luo, L., Zhang, S., Wang, Y., Rahman, M., Syk, I., Zhang, E., Thorlacius, H., 2014. Proinflammatory role of neutrophil extracellular traps in abdominal sepsis. Am. J. Physiol. Lung Cell. Mol. Physiol. 307, L586-L596.

Marques, C.S., Passero, L.F., Vale-Gato, I., Rodrigues, A., Rodrigues, O.R., Martins, C., Correia, I., Tomás, A.M., Alexandre-Pires, G., Ferronha, M.H., Santos-Gomes, G.M., 2015. New insights into neutrophil and Leishmania infantum in vitro immune interactions. Comp. Immunol. Microbiol. Infect. Dis. 40, 19-29.

Matte, C., Olivier, M., 2002. Leishmania-induced cellular recruitment during the early inflammatory response: modulation of proinflammatory mediators. J. Infect. Dis. 185, 673-681.

Pearson, R.D., Steigbigel, R.T., 1981. Phagocytosis and killing of the protozoan Leishmania donovani by human polymorphonuclear leukocytes. J. Immunol. 127, 1438-1443.

Pompeu, M.L., Freitas, L.A., Santos, M.L., Khouri, M., Barral-Netto, M., 1991. Granulocytes in the inflammatory process of BALB/c mice infected by Leishmania amazonensis. A quantitative approach. Acta Trop. 48, 185-193.

Ribeiro-Gomes, F.L., Sacks, D., 2012. The influence of early neutrophil-Leishmania interactions on the host immune response to infection. Front. Cell. Infect. Microbiol. 2, 59. http://dx.doi.org/10.3389/fcimb.2012.00059.

Ribeiro-Gomes, F.L., Peters, N.C., Debrabant, A., Sacks, D.L., 2012. Efficient capture of infected neutrophils by dendritic cells in the skin inhibits the early anti-leishmania response. PLoS Pathog. 8, e1002536. http://dx.doi.org/10.1371/journal.ppat. 1002536.

Rittig, M.G., Bogdan, C., 2000. Leishmania-host-cell interaction: complexities and alternative views. Parasitol. Today 16, 292-297.

Santos-Gomes, G.M., Abranches, P., 1996. Comparative study of infectivity caused by promastigotes of Leishmania infantum MON-1 L. infantumMON-24 and L. donovaniMON-18. Folia Parasitol. (Praha) 43, 7-12.

Santos-Gomes, G.M., Campino, L., Abranches, P., 2000. Canine experimental infection: intradermal inoculation of Leishmania infantum promastigotes. Mem. Inst. Oswaldo Cruz 95, 193-198.

Scaffidi, P., Misteli, T., Bianchi, M.E., 2002. Release of chromatin protein HMGB1 by necrotic cells triggers inflammation. Nature 418, 191-195 Erratum in: Nature. 2010. $467,622$.

Silva, M.T., 2010. Secondary necrosis: the natural outcome of the complete apoptotic program. FEBS Lett. 584, 4491-4499.

Strasser, A., Kalmar, E., Niedermüller, H., 1998. A simple method for the simultaneous separation of peripheral blood mononuclear and polymorphonuclear cells in the dog. Vet. Immunol. Immunopathol. 62, 29-35.

Thalhofer, C.J., Chen, Y., Sudan, B., Love-Homan, L., Wilson, M.E., 2011. Leukocytes infiltrate the skin and draining lymph nodes in response to the protozoan Leishmania infantum chagasi. Infect. Immun. 79, 108-117.

Ueno, N., Wilson, M.E., 2012. Receptor-mediated phagocytosis of Leishmania: implications for intracellular survival. Trends Parasitol. 28, 335-344.

van Zandbergen, G., Hermann, N., Laufs, H., Solbach, W., Laskay, T., 2002. Leishmania promastigotes release a granulocyte chemotactic factor and induce interleukin-8 release but inhibit gamma interferon-inducible protein 10 production by neutrophil granulocytes. Infect. Immun. 70, 4177-4184.

van Zandbergen, G., Klinger, M., Mueller, A., Dannenberg, S., Gebert, A., Solbach, W., Laskay, T., 2004. Cutting edge: neutrophil granulocyte serves as a vector for Leishmania entry into macrophages. J. Immunol. 173, 6521-6525.

Yamamoto, E.S., Campos, B.L., Jesus, J.A., Laurenti, M.D., Ribeiro, S.P., Kallás, E.G., Rafael Fernandes, M., Santos-Gomes, G., Silva, M.S., Sessa, D.P., Lago, J.H., Levy, D., Passero, L.F., 2015. The effect of ursolic acid on Leishmania (Leishmania) amazonensisis related to programed cell death and presents therapeutic potential in experimental cutaneous leishmaniasis. PLoS One 10 (12), e0144946. 